\title{
Semi-empirical determination of the nuclear quadrupole moment of ${ }^{109} \mathrm{Sn}$
}

\author{
M. Elantkowska ${ }^{1, \mathrm{a}} \mathbb{D}$, J. Ruczkowski $^{2}$, S. Wilman $^{1}$, M. Suski ${ }^{1}$ \\ ${ }^{1}$ Institute of Materials Research and Quantum Engineering, Faculty of Materials Engineering and Technical \\ Physics, Poznan University of Technology, Piotrowo 3, 60-965 Poznan, Poland \\ 2 Institute of Robotics and Machine Intelligence, Faculty of Control, Robotics and Electrical Engineering, \\ Poznan University of Technology, Piotrowo 3A, 60-965 Poznan, Poland
}

Received: 19 October 2020 / Accepted: 13 February 2021

(C) The Author(s) 2021

\begin{abstract}
The nuclear quadrupole moment $(Q)$ of ${ }^{109} \mathrm{Sn}$ was determined by means of hyperfine structure $(h f s)$ many-body parametrization method. The hyperfine structure splittings for isotopes ${ }^{117-131} \mathrm{Sn}$ recently measured by Yordanov et al. (Commun Phys 3:1, 2020) were used in multiconfiguration semi-empirical calculations. The contributions from the second-order perturbation theory to the magnetic dipole hyperfine structure, concerning electrostatically correlated hyperfine interactions, were taken into consideration for even and odd configurations simultaneously. Contributions from the second-order perturbation theory to the electric quadrupole hyperfine structure, concerning spin-orbit correlated hyperfine interactions, were included for the first time.
\end{abstract}

\section{Introduction}

The nuclear quadrupole moments $(Q)$ constitute an important feature of nuclei which allow one determination of the deviation of the nuclear charge distribution from a spherical shape. However, the knowledge of the values of nuclear quadrupole moments is important not only in the study of nuclei themselves, but also in molecular and solid state physics, and even in many areas of chemical, biological and medical sciences [2]. The compilations of available $Q$ values were published over the years by Raghavan [3], Stone [2,4] and Pyykkö [5].

In the case of $\mathrm{Sn}$ atom, due to the long chain of the isotopes with particularly interesting semi-magic nuclei, both experimental and theoretical studies of nuclear properties have been conducted for many years [6-12].

Recently, Yordanov et al. [1] reported highly precise values of the quadrupole moments of $Q\left({ }^{109,117-131} \mathrm{Sn}\right)$, which were based on the measured atomic hyperfine structure. The experimental data were acquired by exploiting the collinear laser-induced fluorescence on the ionic beam of mass separated tin isotopes formed into short bunches by means of a linear Paul trap. The credibility of the obtained experimental values of quadrupole moments was further confirmed by the large-scale atomic structure calculations, which exploited the fully relativistic multiconfiguration Dirac-Hartree-Fock (MCDHF) method, using the General Relativistic Atomic Structure Package computer codes (GRASP) [13,14], as well as the

\footnotetext{
a e-mail: magdalena.elantkowska@ put.poznan.pl (corresponding author)
} 
configuration interaction Dirac-Fock-Sturm (CI-DFS) method [15] concerning the dipole hyperfine constants in the triplet $5 \mathrm{~s}^{2} 5 \mathrm{p} 6 \mathrm{~s}^{3} \mathrm{P}_{1}$ state.

Furthermore, the researchers representing leading theoretical groups published together the details of the atomic calculations of the electric field gradient (EFG) values for the $5 \mathrm{~s}^{2} 5 \mathrm{p} 6 \mathrm{~s}$ ${ }^{1,3} \mathrm{P}_{1}$ excited states in neutral tin [16], which were previously presented in Ref. [1]. They used three independent computational strategies of the variational multiconfiguration DiracHartree-Fock method and a fourth approach based on the configuration interaction DiracFock-Sturmian theory; their work also comprises new calculations not included in Ref. [1]. The values of the quadrupole moment obtained in the above-mentioned works are mutually consistent and amount to $Q\left({ }^{109} \mathrm{Sn}\right)=218(7)(15) \mathrm{mb}[1]$ and $Q\left({ }^{109} \mathrm{Sn}\right)=219(7)(16) \mathrm{mb}$ [16], respectively. These values are, however, significantly different from the result obtained by Eberz et al. [17], which amounted to $Q\left({ }^{109} \mathrm{Sn}\right)=310(100) \mathrm{mb}$, and also the re-normalized Eberz's value of 330(110) mb, quoted by Stone in his review work [2]. Since there are no results for quadrupole moments of tin available from measurements on the muonic or pionic atoms and the discrepancy between the recently reported and the formerly accepted value for $Q\left({ }^{109} \mathrm{Sn}\right)$ is ca. one third of the latter, it is very important to determine the independent quadrupole moment's value using methods other than those presented in the recent works of Yordanow and Papoulia [1,16]. Therefore, we decided to join the research aimed at resolving the above-mentioned discrepancies, using a different method, independent of the value of the electric field gradient.

For many years, we have developed a software package dedicated for comprehensive semi-empirical analysis of the fine and hyperfine structure, as well as transition probabilities, for complex atoms. It allows one to determine the most accurate possible wave functions describing the state of an atom and, consequently, to determine the predicted values of the attributes of the atomic structure in accordance with the rules of quantum mechanics. In our opinion, this method, due to its iterative character, allows the control of compliance of the results with the experiment and gives the opportunity to describe the structure of the atom and the processes occurring therein.

In 2020, we published the first comprehensive semi-empirical analysis of the odd-parity configuration system of atomic tin [18]. Due to the very good compliance between the calculated and the experimental values of the levels' energies, the obtained wave functions could be successfully used in the analysis of the data concerning radiative transitions for Sn I, available in the literature. After application of our own method of oscillator strengths parametrization, described in works [19-21], the values of radiative parameters for transitions from the odd-parity levels in a wide spectral range, as well as radiative lifetimes of the excited levels, were predicted. Thus, we have an opportunity to use the latest data on the $h f s$ splittings of Sn electronic levels [1] and our results of the semi-empirical fine-structure calculations [18] for the purpose of many-body hyperfine structure parametrization and determine an independent value of the nuclear quadrupole moment of ${ }^{109} \mathrm{Sn}$.

In Sect. 2, we describe in detail the calculations of the fine structure for both odd and even configurations of atomic tin, using our method for semi-empirical analysis of complex electronic systems in multiconfiguration approximation up to the second order of the perturbation theory. Section 3 contains a detailed description of parametrization of the hyperfine interactions, including electrostatically correlated hyperfine interactions and spin-orbit correlated hyperfine interactions. The results of semi-empirical calculations for even and odd configuration systems of the tin atom, used for determination of the nuclear quadrupole moment of ${ }^{109} \mathrm{Sn}$, are presented in Sect. 4. In Appendix, explicit formulae for spin-orbit correlated hyperfine interactions are presented. 


\section{Semi-empirical analysis of the fine structure}

The original method of the fine structure parametrization, presented by us in detail in papers [22-28], allows one obtaining the wave functions describing the electron states. Verification of the correctness of these wave functions can be carried out by means of the parametrization of the hyperfine structure or radiative properties.

In the framework of the published paper [18], we performed large-scale semi-empirical calculations of 184 odd-parity configurations: $5 \mathrm{~s} 5 \mathrm{p}^{3}, 5 \mathrm{p}^{3} 5 \mathrm{~d}, 5 \mathrm{p}^{3} 5 \mathrm{~g}, 5 \mathrm{~s} 5 \mathrm{p}^{2} n^{\prime} \mathrm{p}\left(n^{\prime}=6-15\right)$, $5 \mathrm{~s} 5 \mathrm{p}^{2} n^{\prime} \mathrm{f}\left(n^{\prime}=4-13\right), 5 \mathrm{~s} 5 \mathrm{p} 6 \mathrm{~s}^{2}, 5 \mathrm{~s} 5 \mathrm{p} 5 \mathrm{~d}^{2}, 5 \mathrm{~s}^{2} 5 \mathrm{p} n^{\prime} \mathrm{s}\left(n^{\prime}=6-80\right), 5 \mathrm{~s}^{2} 5 \mathrm{p} n^{\prime} \mathrm{d}\left(n^{\prime}=5-72\right)$, $5 \mathrm{~s}^{2} 5 \mathrm{p} n^{\prime} \mathrm{g}\left(n^{\prime}=5-20\right)$. In the least-squares fitting procedure for 646 experimentally known energy levels, the mean deviation between calculated and experimental values of $11 \mathrm{~cm}^{-1}$ was achieved. For the worst-matched level, the relative deviation of the calculated value from the experimental one amounts to ca. $0.13 \%$.

Only a small excerpt from the results is given in Table 1 ; the entire results of the $f s$ calculations: the values of calculated energies of the electronic levels and the values of the fine structure radial parameters, are presented in Tables I, II, III in [18].

Since the experimental data of the magnetic-dipole hyperfine structure constants $A$ are available only for the levels of the lowest $5 \mathrm{~s}^{2} 5 \mathrm{p}^{2}$ configuration, we considered a limited system of 7 even-parity configurations: $5 \mathrm{~s}^{2} 5 \mathrm{p}^{2}, 5 \mathrm{~s}^{2} 5 \mathrm{p} n^{\prime} \mathrm{p}\left(n^{\prime}=6-8\right), 5 \mathrm{~s}^{2} 5 \mathrm{p} n^{\prime} \mathrm{f}\left(n^{\prime}=4-6\right)$. This configuration system was chosen in order to take into account the admixture to the $5 \mathrm{~s}^{2} 5 \mathrm{p}^{2}$ levels eigenvector. A small part of the results of the fine structure calculations of the even-parity configuration system is presented in Table 2.

After diagonalization of the fine structure energy matrix, the values of the radial parameters describing the fine structure and the wave functions were obtained. The next step of the calculation procedures, taking into account the wave functions derived from the fine structure analysis, allows one to obtain linear equations describing experimental hyperfine structure constants. The solution of an overdetermined system of equations, by means of the least squares method, enables obtaining radial parameters and expected hyperfine structure constants. The scheme of the main calculation procedures used in the fine and hyperfine structure analysis was outlined in Ref. [24].

\section{Hyperfine structure many-body parametrization method}

Sandars and Beck [29] constructed the effective-operator formalism required for the relativistic hyperfine interaction in complex atoms. The theory simplifies the calculations in their treatment of the magnetic dipole and electric quadrupole interaction as well as the interpretation of relativistic $h f s$ effects in many-electron atoms. Each of the multipole interaction is described by three effective radial parameters for each open electronic shell. For atoms with many electrons in an open shell, the magnetic dipole and the electric quadrupole hyperfine interactions are commonly analysed using the one-electron radial parameters usually defined as:

$$
\begin{aligned}
& a_{n l}^{\kappa k}=\frac{\mu_{0}}{4 \pi} \frac{2 \mu_{B}}{h} \frac{\mu_{I}}{I}\left\langle\left. r^{-3}\right|_{n l} ^{\kappa k} \quad \kappa k=01,12,\right. \\
& a_{n l}^{\kappa k}=\frac{\mu_{0}}{4 \pi} \frac{2 \mu_{B}}{h} \frac{\mu_{I}}{I} \frac{2}{3}\left\langle\left. r^{-3}\right|_{n l} ^{\kappa k} \quad \kappa k=10,\right. \\
& b_{n l}^{\kappa k}=\frac{1}{4 \pi \epsilon_{0}} \frac{e^{2}}{h} Q\left\langle\left. r^{-3}\right|_{n l} ^{\kappa k} \quad \kappa k=02,13,11,\right.
\end{aligned}
$$




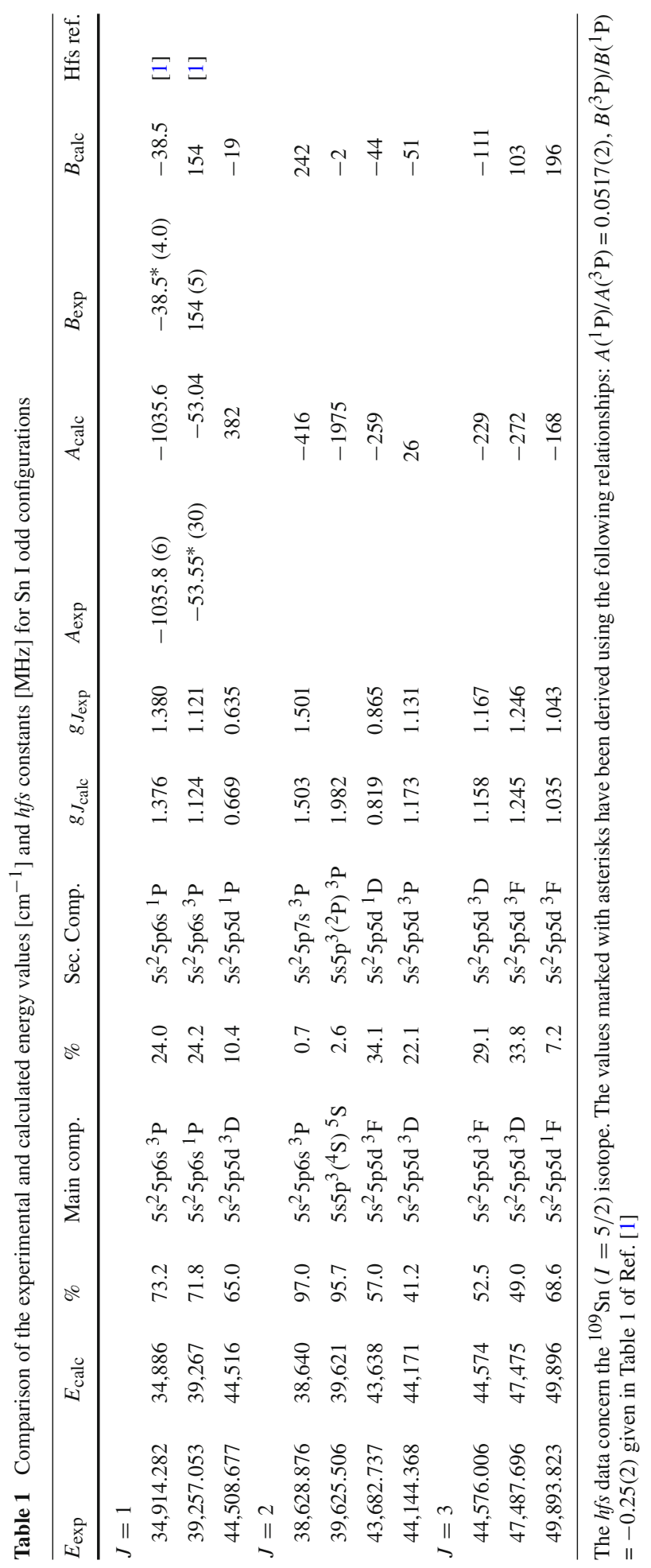




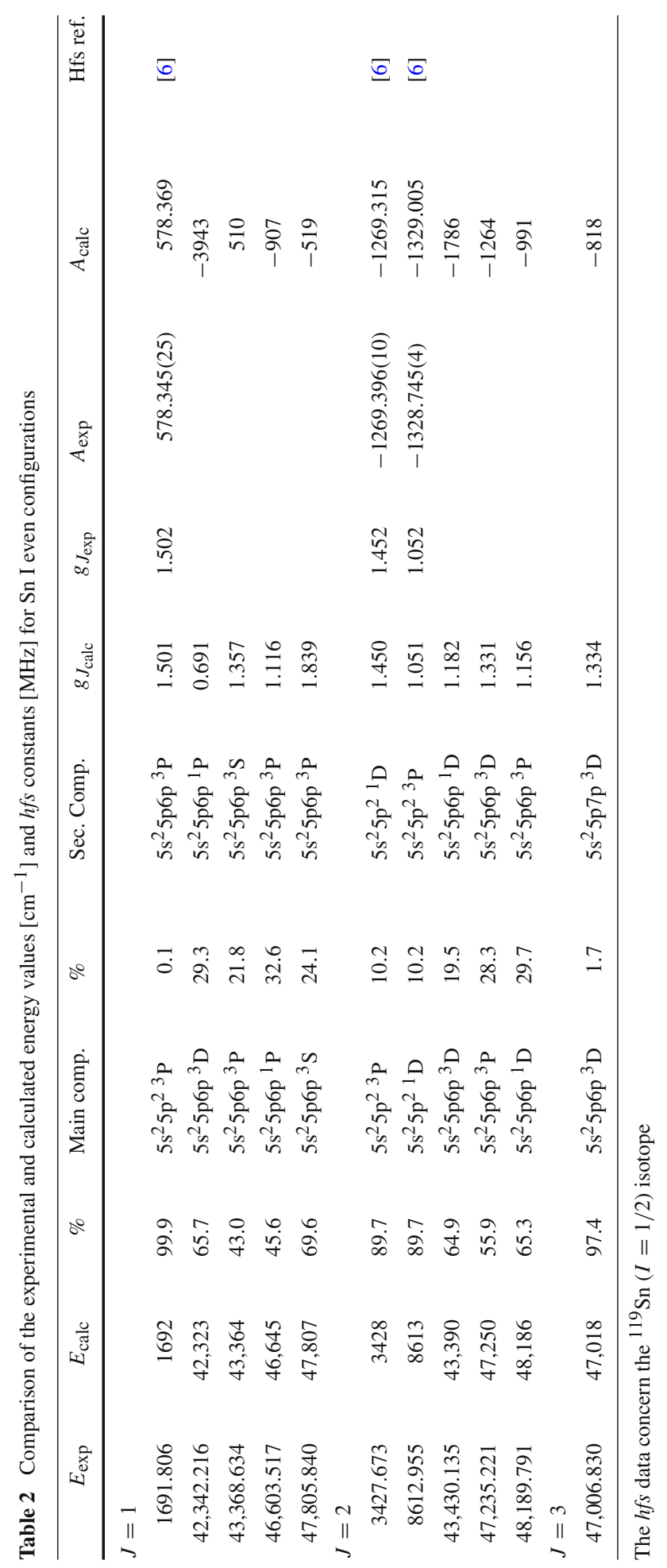


where $a^{01}$ is related to the interaction between the nuclear magnetic-dipole moment $\mu_{I}$ and the magnetic field produced by solely orbital motion of the electrons, $a^{12}$ represents the interaction between $\mu_{I}$ and the magnetic moment of the electron, $a^{10}$ is related to the relativistic and configuration interaction (core polarization effects), $b^{02}$ represents the electrostatic interaction between the nucleus and those electron configurations that have a spherically symmetric charge distribution which is finite at the nucleus, and $b^{13}, b^{11}$ are purely relativistic in nature.

It is a common knowledge that corrections from a single distant perturbing configurations are rather small, but their cumulative effect may be considerable, because of increasing density of states as the continuum is approached; that's why the second-order effects, so-called configuration interaction effects (CI), should also be considered in the hyperfine structure study using an effective Hamiltonian of Sandars and Beck [29]. The method of parametrization of the configuration interaction effects in the hyperfine structure was described by us in [30]. We showed that, using the available experimental data, it was possible to quantitatively determine two-body contributions to the hyperfine structure, originating from the excitations from electronic closed shells to open shells and from open shells to empty shells.

Contributions from the second-order perturbation theory to electrostatically correlated hyperfine interactions (CHFS) are specified as follows:

$$
\mathbf{C H F S}=-\sum_{\psi^{\prime \prime} \neq \psi, \psi^{\prime}}\left[\left\langle\psi|\mathbf{G}| \psi^{\prime \prime}\right\rangle \times\left\langle\psi^{\prime \prime}\left|\mathbf{T}^{(\kappa k) K}\right| \psi^{\prime}\right\rangle+\left\langle\psi\left|\mathbf{T}^{(\kappa k) K}\right| \psi^{\prime \prime}\right\rangle \times\left\langle\psi^{\prime \prime}|\mathbf{G}| \psi^{\prime}\right\rangle\right] / \Delta E,
$$

where $\mathbf{G}$ is a two-body electrostatic interaction operator, $\mathbf{T}^{(\kappa k) K}$ a one-body operator; the latter may represent the hyperfine $\left(\mathbf{H}_{\mathrm{hfs}}\right)$ interaction, $\psi, \psi^{\prime}$ represent particular states of the considered system of configurations, $\psi^{\prime \prime}$ denote all perturbing virtual states taken into account in our system, and $\Delta E$ denotes the energy difference between the centre of gravity of the considered configuration and the particular perturbing configuration.

Details of corrections from the second-order perturbation theory for electrostatically correlated hyperfine interactions were presented in our earlier work [28]. The most often discussed and analysed effect is the one regarding the excitation of electrons from the closed shells $n_{0}$ s to the empty shells $n$ s. In Sandars and Beck theory [29], the radial parameters $b_{n l}^{11}$ and $b_{n l}^{13}$ represent relativistic effects in the hyperfine structure. So, like for the radial parameter $a_{n l}^{10}$ of the magnetic dipole interaction, the above-mentioned second-order contributions to the electric quadrupole interaction and relativistic effects are impossible to separate and cannot be regardless designated in the least-squares procedure. For that reason, in our approach $a_{n l}^{10}=0$ for $l>0$ is adopted. The so-called core polarization effects in the hyperfine structure were considered by us in detail in a separate work [31].

The scarcity of hyperfine structure constants of atomic tin means that it is impossible to avoid all linear dependencies in both $A$ and $B$ equation systems of the many-body hyperfine structure parametrization method. Wherefore, additional presumptions from the fine structure analysis had to be taken into account in our $h f s$-fitting procedure. Eigenvectors concerning the states of the configuration space include contributions originated from the configurations with $\mathrm{p}$ and d electrons. Therefore, the contribution from the $\mathrm{p}$ and delectrons to the $B$ electric quadrupole constants was defined by the relation: $\zeta_{5 p} / \zeta_{5 d}=b_{5 p}^{02} / b_{5 d}^{02}$.

The values of spin-orbit parameters were accurately derived from our earlier many-body fine structure parametrization in an extended model space of 184 configurations. Contributions from the electrostatically correlated spin-orbit interaction (CSO) were taken into account. The operators of electrostatically correlated spin-orbit interactions and electrostatically correlated $h f s$ interactions have the same structure; consequently, an analogy can be 
made between CSO and CHFS. Due to the fact that they both include one-electron operators $\left(\mathbf{H}_{\mathrm{hfs}}, \mathbf{H}_{\mathrm{so}}\right)$, exactly the same one-electron excitations are accountable for these second-order effects, i.e. we can parameterize the above effects analogously. It follows from the above that we can assume the relations:

$\frac{\mathrm{D}^{t}\left(n_{0} l_{0} n l, n l n l\right) \zeta_{n_{0} l_{0}, n l}}{\zeta_{n l}} \approx \frac{\mathrm{D}^{t}\left(n_{0} l_{0} n l, n l n l\right) \mathrm{P}^{\kappa k}\left(n_{0} l_{0}, n l\right)}{b_{n l}^{\kappa k}} \approx \frac{\mathrm{D}^{t}\left(n_{0} l_{0} n l, n l n l\right) \mathrm{P}^{\kappa k}\left(n_{0} l_{0}, n l\right)}{a_{n l}^{\kappa k}}$,

where $t=0,2,4, n l=5 \mathrm{p}$ or $n l=5 \mathrm{~d}$, and $\kappa k=01,12$ and 02 . In the fitting procedure, $a_{n l}^{\kappa k}$ and $b_{n l}^{\kappa k}$ parameters are determined, the same for all the aforementioned configurations.

Within this work, contributions from the second-order perturbation theory spin-orbit correlated hyperfine interactions (SO-HFS) were considered for the first time in our procedure:

$$
\begin{aligned}
\text { SO-HFS }= & -\sum_{\psi^{\prime \prime} \neq \psi, \psi^{\prime}}\left[\left\langle\psi\left|\mathbf{H}_{\mathrm{so}}\right| \psi^{\prime \prime}\right\rangle \times\left\langle\psi^{\prime \prime}\left|\mathbf{H}_{\mathrm{hfs}}\right| \psi^{\prime}\right\rangle\right. \\
& \left.+\left\langle\psi\left|\mathbf{H}_{\mathrm{hfs}}\right| \psi^{\prime \prime}\right\rangle \times\left\langle\psi^{\prime \prime}\left|\mathbf{H}_{\mathrm{so}}\right| \psi^{\prime}\right\rangle\right] / \Delta E,
\end{aligned}
$$

where $\mathbf{H}_{\text {so }}$ stands for the one-body operator of the spin-orbit interaction, and the other notations are the same as in Eq. (2). This perturbation effects were mentioned for the first time by Feneuille and Armstrong in their work concerning the additive nature of correlation and relativistic effects in $h f s$ [32]. They wrote about the hierarchy of changes induced by assorted terms in the electric-quadrupole interaction. The CHFS term also changes $b_{n l}^{02}$ but does not affect $b_{n l}^{11}$ or $b_{n l}^{13}$. On the other hand, the SO-HFS term changes $b_{n l}^{11}$ and $b_{n l}^{13}$ but causes no further change in $b_{n l}^{02}$. In Sandars and Beck theory [29], the radial parameters $b_{n l}^{11}$ and $b_{n l}^{13}$ represent relativistic effects in the hyperfine structure. Thus, according to the works $[33,34]$, the above-mentioned effects are impossible to separate and cannot be regardless designated in the least-squares procedure like for magnetic dipole interactions. Therefore, in our procedure $b_{5 p}^{11}=0$ was assumed, and the second-order parameter $\mathrm{P}^{11}\left(5 \mathrm{p}, n^{\prime} \mathrm{p}\right) \zeta\left(5 \mathrm{p}, n^{\prime} \mathrm{p}\right)$ was fitted.

The situation is more complicated when we investigate the magnetic-dipole interaction. The parameters $a_{n l}^{01}$ and $a_{n l}^{12}$ are modified by the second-order spin-orbit term. The changes can be described by two parts of contribution to the radial parameters: an independent one and a dependent one. In our parametrization method, contributions from spin-orbit correlated hyperfine interactions and electrostatically correlated hyperfine interactions are linearly dependent and could not be used simultaneously in the fitting procedure.

In order to perform the hyperfine structure parametrization for magnetic-dipole interaction, we used three $h f s$ constants $A$ for ${ }^{3} \mathrm{P}_{1,2}$ and ${ }^{1} \mathrm{D}_{2} 5 \mathrm{~s}^{2} 5 \mathrm{p}^{2}$ states of ${ }^{119} \mathrm{Sn}$ measured by Childs using the atomic-beam magnetic resonance technique [6], adequately recalculated (see below), and two $h f s$ constants for ${ }^{3} \mathrm{P}_{1}$ and ${ }^{1} \mathrm{P}_{1} 5 \mathrm{~s}^{2} 5 \mathrm{p} 6 \mathrm{~s}$ states of ${ }^{109} \mathrm{Sn}$ from Yordanov [1]. For our calculations, the corrected values of the $A$ constants presented in Table III [6] were used to account for disturbances caused by other atomic states.

Due to a very small number of the experimental hyperfine constants available, it was proposed to parameterize the hyperfine structure interaction for both configuration parities simultaneously.

Calculations of the magnetic-dipole moment for the unstable $\mathrm{Sn}$ isotopes are straightforward with the data of ${ }^{119} \mathrm{Sn}$ as in reference [17]:

$$
\mu_{I}^{x}=\mu_{I}^{119}\left(I^{x} / I^{119}\right) \times\left(A^{x} / A^{119}\right) .
$$

Inserting into the above relation the following values: $\mu_{I}\left({ }^{119} \mathrm{Sn}\right)=-1.04728(7) \mu_{N}$ for $I=1 / 2$, corrected for diamagnetism [3], $A\left(5 \mathrm{p} 6 \mathrm{~s}{ }^{3} \mathrm{P}_{1},{ }^{119} \mathrm{Sn}(I=1 / 2)\right)=-5007.95(17)$ 
[7] and $A\left(5 \mathrm{p} 6 \mathrm{~s}{ }^{3} \mathrm{P}_{1},{ }^{109} \mathrm{Sn}(I=5 / 2)\right)=-1035.8(6)$ [1], yields a magnetic moment of $\mu_{I}\left({ }^{109} \mathrm{Sn}\right)=-1.08305(74) \mu_{N}$. Although several papers indicated the need for revision of the nuclear magnetic-dipole moments of tin, suggesting corrections of the order of $0.2 \%$ [3538] compared to the hitherto accepted values [3], this change does not affect our calculation results as part of the accuracy of our method.

Based on the value $\mu_{I}\left({ }^{109} \mathrm{Sn}\right)=-1.08305(74) \mu_{N}$, the corrected values of constants $A$ for the $5 \mathrm{p}^{2}{ }^{3} \mathrm{P}_{1,2},{ }^{1} \mathrm{D}_{2}$ states of ${ }^{119} \mathrm{Sn}(I=1 / 2)$ published by Childs [6], were recalculated as follows:

$$
A^{109}=A^{119}\left(I^{119} / I^{109}\right) \times\left(\mu_{I}^{109} / \mu_{I}^{119}\right),
$$

and they amount to $A\left({ }^{3} \mathrm{P}_{1},{ }^{109} \mathrm{Sn}\right)=119.620(95) \mathrm{MHz}, A\left({ }^{3} \mathrm{P}_{2},{ }^{109} \mathrm{Sn}\right)=-262.55(20) \mathrm{MHz}$ and $A\left({ }^{1} \mathrm{D}_{2},{ }^{109} \mathrm{Sn}\right)=-274.83(20) \mathrm{MHz}$, respectively.

In the case of the magnetic-dipole $h f s$ interactions, the parametrization was performed for even and odd configurations simultaneously. The hyperfine structure parametrization for the electric-quadrupole $h f s$ interactions was conducted for odd-parity configurations only because of the lack of any experimental data for the opposite parity. The use of the parameters describing spin-orbit contributions to the hyperfine structure interaction originating from the second-order perturbation theory properly described the electric quadrupole interaction. The assumed relationship $\mathrm{P}^{11}\left(5 \mathrm{p}, n^{\prime} \mathrm{p}\right) \zeta\left(5 \mathrm{p}, n^{\prime} \mathrm{p}\right) / b_{5 p}^{02}=0.3179$, concluded from the analysis of the first and the second-order radial integrals obtained in the fine structure calculations, is consistent with predictions of Feneuille and Armstrong [32]. The authors wrote that the above ratio may be of a similar absolute value but of the opposite sign as the ratio $\left\langle\left. r^{-3}\right|_{5 p} ^{11} /\left\langle\left. r^{-3}\right|_{5 p} ^{02}\right.\right.$. This ratio was determined by Lindgren and Rosen [39] and amounts to $\left\langle\left. r^{-3}\right|_{5 p} ^{11} /\left\langle\left. r^{-3}\right|_{5 p} ^{02}=\right.\right.$ -0.2029 .

The results of $h f s$ analysis are presented in Tables 1 and 2 together with our earlier results of semi-empirical $f s$ calculations. The experimental and calculated values of levels' energies are listed in first two columns, respectively. The following four columns contain the strongest and second strongest $f s$ components with the corresponding percentages. The next two columns contain information about the calculated and experimental $g_{J}$ values. The experimental and calculated hyperfine constants are presented in the next columns. In the last column, the references to experimental $h f s$ constants are given.

The values of one- and two-body hyperfine structure radial parameters are presented in Table 3 .

\section{Determination of the nuclear quadrupole moment}

Applying the effective $h f s$ parameters $a_{5 p}^{01}, b_{5 p}^{02}$ from the $h f s$ parametrization procedure presented above, one can determine the nuclear quadrupole moment $Q$, which should be almost free from Sternheimer effects up to the second order of perturbation theory:

$$
Q=\frac{2 \mu_{B} g_{I}}{e^{2}} \frac{b_{5 p}^{02}}{a_{5 p}^{01}}=(220 \pm 8) \mathrm{mb},
$$

where $g_{I}$ is the nuclear $g_{I}$-factor expressed in nuclear magnetons (for ${ }^{109} \mathrm{Sn}(I=5 / 2)$ nucleus $\left.g_{I}=-0.43322\right)$; the values of the parameters $a_{5 p}^{01}=-566(1)$ and $b_{5 p}^{02}=708(27)$ are given in $\mathrm{MHz}$. 


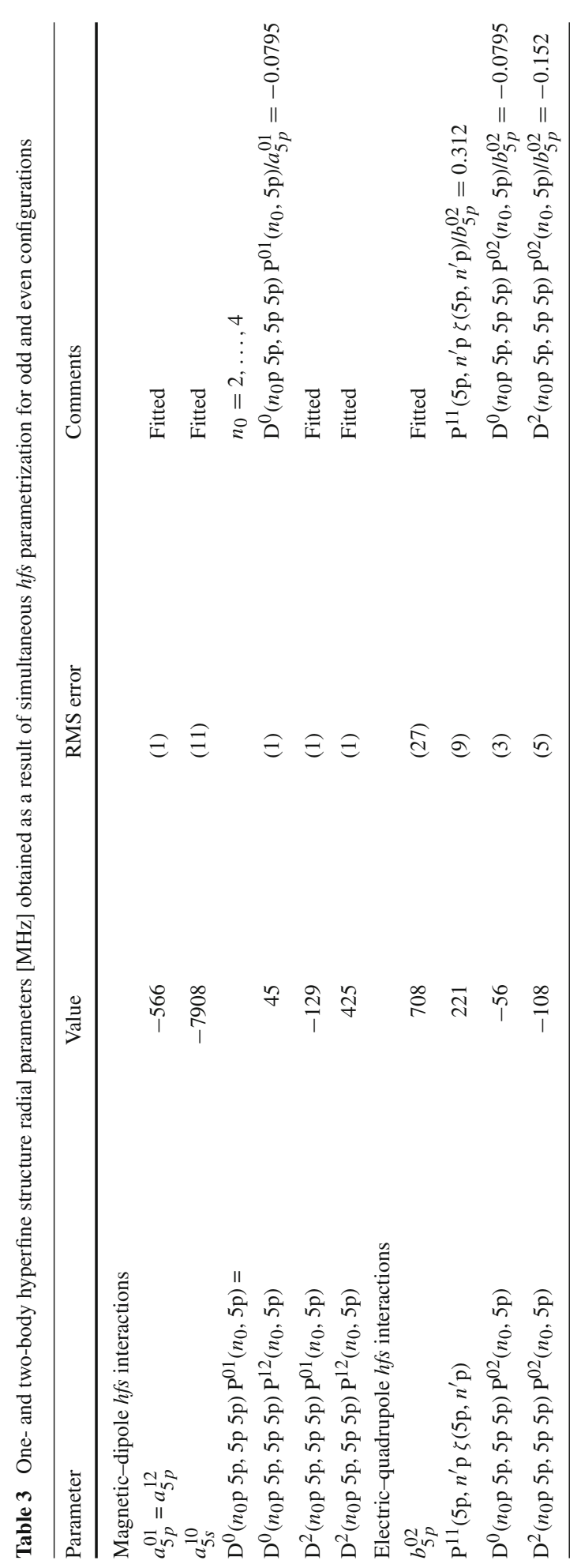


It should be emphasized that in the procedure of determination of the nuclear quadrupole moment of ${ }^{109} \mathrm{Sn}$, all possible contributions resulting from the second-order perturbation theory to the hyperfine structure were taken into consideration for the first time, namely the contributions from electrostatically correlated hyperfine interactions, as well as for spinorbit correlated hyperfine interactions. Therefore, the corrections related to the distortion of inner closed electron shells, referred to Sternheimer shielding (or anti-shielding) factors, are correctly included in the calculations. The value is in excellent concordance with those reported by Yordanov et al. $\left(Q\left({ }^{109} \mathrm{Sn}\right)=218(7)(15) \mathrm{mb}[1]\right.$ and Papoulia et al. $Q\left({ }^{109} \mathrm{Sn}\right)=219(7)(16) \mathrm{mb}$ [16]. Furthermore, the values of the quadrupole moments for other $\mathrm{Sn}$ isotopes, which can be determined from the currently available experimental data and relevant relationships, would be consistent with the values given in Table 1 in Ref. [1].

\section{Conclusions}

We determined the value of the nuclear quadrupole moment of ${ }^{109} \mathrm{Sn}$ by means of a multiconfiguration semi-empirical parametrization of the hyperfine structure. The achievement of the results was possible, since the precise eigenfunctions describing the electronic states were known. Our procedures for the description of the structure of a complex atom allow for effective coupling between the calculations and the experiment, and thus, new experimental data allow to improve the calculated predictions.

The method presented by us can be used to determine the nuclear quadrupole moments of other elements and contribute to the solution of the issues where the values available so far, such as, for example, for ${ }^{209} \mathrm{Bi}$, are divergent [5,40,41].

Acknowledgements The authors would like to express their gratitude to Dr. Sci. Danuta Stefańska for her valuable remarks and suggestions. Financial support of this work by the Ministry of Science and Higher Education, Poland, within the projects realized at Poznan University of Technology: 0511/SBAD/2053 at Faculty of Materials Engineering and Technical Physics, (M.E., S.W. and M.S.) and 0214/SBAD/0210 at Institute of Robotics and Machine Intelligence (J.R.) is greatly acknowledged.

Open Access This article is licensed under a Creative Commons Attribution 4.0 International License, which permits use, sharing, adaptation, distribution and reproduction in any medium or format, as long as you give appropriate credit to the original author(s) and the source, provide a link to the Creative Commons licence, and indicate if changes were made. The images or other third party material in this article are included in the article's Creative Commons licence, unless indicated otherwise in a credit line to the material. If material is not included in the article's Creative Commons licence and your intended use is not permitted by statutory regulation or exceeds the permitted use, you will need to obtain permission directly from the copyright holder. To view a copy of this licence, visit http://creativecommons.org/licenses/by/4.0/.

\section{A Explicit formulae for configuration interaction effects}

\section{A.1 Removal of the $J$-dependence and the method of the reduced matrix elements calculation}

The formulae describing the intraconfiguration spin-orbit correlated hyperfine interaction are written as the reduced matrix elements. Each two-body contribution to the spin-orbit parameter and to the $h f s$ magnetic-dipole and electric-quadrupole constants can be expressed as a reduced matrix element using the Wigner-Eckart theorem. For $n j$-coefficients, oneparticle fractional parentage coefficients, the generally accepted notations were used. In this 
work, we derived explicit expressions for $n l^{N}, n l^{N} n_{1} l_{1}^{N_{1}}$ and $n l^{N} n_{1} l_{1}^{N_{1}} n_{2} l_{2}$ configurations taking into account the excitation of one electron from an open shell to an empty shell. Contributions from the second-order perturbation theory for CHFS are given by:

$$
\begin{aligned}
& \langle\Psi(\Gamma \alpha S L J M)| \text { SO-HFS }\left|\Psi^{\prime}\left(\Gamma^{\prime} \alpha^{\prime} S^{\prime} L^{\prime} J^{\prime} M^{\prime}\right)\right\rangle \\
& =-\sum_{\psi^{\prime \prime} \neq \psi, \psi^{\prime}}\left[\left\langle\psi\left|\mathbf{H}_{\mathrm{so}}{ }^{(11) 0}\right| \psi^{\prime \prime}\right\rangle \times\left\langle\psi^{\prime \prime}\left|\mathbf{T}^{(\kappa k) K}\right| \psi^{\prime}\right\rangle\right. \\
& \left.\quad+\left\langle\psi\left|\mathbf{T}^{(\kappa k) K}\right| \psi^{\prime \prime}\right\rangle \times\left\langle\psi^{\prime \prime}\left|\mathbf{H}_{\mathrm{so}}{ }^{(11) 0}\right| \psi^{\prime}\right\rangle\right] / \Delta E= \\
& =-(\text { angular part }) \times(\text { radial part }),
\end{aligned}
$$

where $\psi, \psi^{\prime}$ denote particular states of the considered system of configurations and $\psi^{\prime \prime}$ denote possible perturbing virtual states included in considered system, defined by the formulae.

By substituting $K=2$ and $\kappa k=11$ or 13 into Eq. (8) for electric quadrupole interactions, general formula for the matrix elements of SO-HFS reads:

$$
\begin{aligned}
& \langle\Psi(\Gamma \alpha S L J M)| \text { SO-HFS }\left|\Psi^{\prime}\left(\Gamma^{\prime} \alpha^{\prime} S^{\prime} L^{\prime} J^{\prime} M^{\prime}\right)\right\rangle \\
& =\delta\left(M, M^{\prime}\right) \delta\left(J, J^{\prime}\right)(-1)^{J+S^{\prime}+L}\left\{\begin{array}{lll}
S & S^{\prime} & 1 \\
L & L^{\prime} & J
\end{array}\right\} \sqrt{\frac{20 J(2 J+1)(2 J-1)}{(J+1)(2 J+3)}} \\
& \left\{\begin{array}{lll}
S & S^{\prime} & \kappa \\
L & L^{\prime} & k \\
J & J & 2
\end{array}\right\}\left\langle\Psi(\Gamma \alpha S L)\|\mathbf{S O}-\mathbf{H F S}\| \Psi^{\prime}\left(\Gamma^{\prime} \alpha^{\prime} S^{\prime} L^{\prime}\right)\right\rangle \\
& =\delta\left(M, M^{\prime}\right) \delta\left(J, J^{\prime}\right)(-1)^{J+S^{\prime}+L}\left\{\begin{array}{lll}
S & S^{\prime} & 1 \\
L & L^{\prime} & J
\end{array}\right\} \sqrt{\frac{20 J(2 J+1)(2 J-1)}{(J+1)(2 J+3)}}\left\{\begin{array}{lll}
S & S^{\prime} & \kappa \\
L & L^{\prime} & k \\
J & J & 2
\end{array}\right\} \\
& \times\left[-\sum_{\psi^{\prime \prime}}\left\langle\Gamma \alpha S L\left\|\mathbf{H}_{\mathrm{so}}\right\| \Gamma^{\prime \prime} \alpha^{\prime \prime} S^{\prime \prime} L^{\prime \prime}\right\rangle \times\left\langle\Gamma^{\prime \prime} \alpha^{\prime \prime} S^{\prime \prime} L^{\prime \prime}\left\|\mathbf{t}^{\kappa k}\right\| \Gamma^{\prime} \alpha^{\prime} S^{\prime} L^{\prime}\right\rangle+\right. \\
& \left.-\sum_{\psi^{\prime \prime}}\left\langle\Gamma \alpha S L \| \mathbf{t}^{\kappa k \|} \Gamma^{\prime \prime} \alpha^{\prime \prime} S^{\prime \prime} L^{\prime \prime}\right\rangle \times\left\langle\Gamma^{\prime \prime} \alpha^{\prime \prime} S^{\prime \prime} L^{\prime \prime}\left\|\mathbf{H}_{\mathrm{so}}\right\| \Gamma^{\prime} \alpha^{\prime} S^{\prime} L^{\prime}\right\rangle\right] \\
& =\delta\left(M, M^{\prime}\right) \delta\left(J, J^{\prime}\right)(-1)^{J+S^{\prime}+L}\left\{\begin{array}{lll}
S & S^{\prime} & 1 \\
L & L^{\prime} & J
\end{array}\right\} \sqrt{\frac{20 J(2 J+1)(2 J-1)}{(J+1)(2 J+3)}}\left\{\begin{array}{lll}
S & S^{\prime} & \kappa \\
L & L^{\prime} & k \\
J & J & 2
\end{array}\right\} \delta\left(l_{i}, l_{i}^{\prime \prime}\right) \\
& \times(\text { angular part }) \sum_{n_{i}^{\prime \prime}} \zeta_{n_{i} l_{i}, n_{i}^{\prime \prime} l_{i}}\left\langle n_{i} l_{i}\left|r^{-3}\right| n_{i}^{\prime \prime} l_{i}\right\rangle^{\kappa k} / \Delta E,
\end{aligned}
$$

where $\Gamma, \Gamma^{\prime}$ designate configurations under study, $\Gamma^{\prime \prime}$ denote possible perturbing virtual states included in considered system, and $\Delta E$ is the (positive) energy difference between the relevant open and empty-shell orbitals.

The spin-orbit operator $\widehat{s_{i}} \widehat{l_{i}}$ and the $h f s$ operator $\mathbf{t}^{\kappa k}$ for $\kappa k=11,13$ are defined as:

$$
\begin{aligned}
& \left\langle n_{i} l_{i}\left\|\widehat{s_{i}} \widehat{l_{i}}\right\| n_{i}^{\prime \prime} l_{i}^{\prime \prime}\right\rangle=\frac{3}{2} \sqrt{l(l+1)(2 l+1)} \zeta_{n_{i} l_{i}, n_{i}^{\prime \prime} l_{i}} \\
& \left\langle n_{i} l_{i}\left\|t^{\kappa k}\right\| n_{i}^{\prime \prime} l_{i}^{\prime \prime}\right\rangle=\delta\left(l_{i}, l_{i}^{\prime \prime}\right) \sqrt{3 / 10}\left\langle n_{i} l_{i}\left|r^{-3}\right| n_{i}^{\prime \prime} l_{i}\right\rangle^{\kappa k},
\end{aligned}
$$

where $\zeta\left(n_{i} l_{i}, n_{i}^{\prime \prime} l_{i}\right)$ and $\left\langle n_{0} l_{0}\left|r^{-3}\right| n_{i} l_{i}\right\rangle^{\kappa k}$ are the radial parts of above operators. 
A.2 $n l^{N}$ configuration

The states $\psi$ and $\psi^{\prime}$ for $n l^{N}$ configuration are defined as follows: $\psi=n l^{N} \alpha S L, \psi^{\prime}=$ $n l^{N} \alpha^{\prime} S^{\prime} L^{\prime}$.

In this case, the perturbing virtual states are defined as $\psi^{\prime \prime}=n l^{N-1} \alpha_{0}^{\prime \prime} S_{0}^{\prime \prime} L_{0}^{\prime \prime}, n^{\prime \prime} l^{\prime \prime} ; S^{\prime \prime} L^{\prime \prime}$ and $\kappa k=11$ or 13 .

$$
\begin{aligned}
& -\sum_{\psi^{\prime \prime}}\left[\left\langle\psi\left\|\mathbf{H}_{\text {so }}\right\| \psi^{\prime \prime}\right\rangle \times\left\langle\psi^{\prime \prime}\left\|\mathbf{t}^{\kappa k}\right\| \psi^{\prime}\right\rangle+\left\langle\psi\left\|\mathbf{t}^{\kappa k \|}\right\| \psi^{\prime \prime}\right\rangle \times\left\langle\psi^{\prime \prime}\left\|\mathbf{H}_{\text {so }}\right\| \psi^{\prime}\right\rangle\right] / \Delta E \\
& =\sum_{\alpha_{0}^{\prime \prime} S_{0}^{\prime \prime} L_{0}^{\prime \prime}, S^{\prime \prime} L^{\prime \prime}} \delta\left(l, l^{\prime \prime}\right) N\left(n l ^ { N } \alpha S L \{ | n l ^ { N - 1 } \alpha _ { 0 } ^ { \prime \prime } S _ { 0 } ^ { \prime \prime } L _ { 0 } ^ { \prime \prime } ) \left(n l^{N} \alpha^{\prime} S^{\prime} L^{\prime}\left\{\mid n l^{N-1} \alpha_{0}^{\prime \prime} S_{0}^{\prime \prime} L_{0}^{\prime \prime}\right)\right.\right. \\
& {\left[S, L, S^{\prime}, L^{\prime}\right]^{1 / 2}\left[S^{\prime \prime}, L^{\prime \prime}\right] \times\left[\left\{\begin{array}{ccc}
S & S^{\prime \prime} & 1 \\
\frac{1}{2} & \frac{1}{2} & S_{0}^{\prime \prime}
\end{array}\right\}\left\{\begin{array}{ccc}
L & L^{\prime \prime} & 1 \\
l & l & L_{0}^{\prime \prime}
\end{array}\right\}\left\{\begin{array}{ccc}
S^{\prime \prime} & S^{\prime} & \kappa \\
\frac{1}{2} & \frac{1}{2} & S_{0}^{\prime \prime}
\end{array}\right\}\left\{\begin{array}{ccc}
L^{\prime \prime} & L^{\prime} & k \\
l & l & L_{0}^{\prime \prime}
\end{array}\right\}+\left\{\begin{array}{ccc}
S^{\prime} & S^{\prime \prime} & 1 \\
\frac{1}{2} & \frac{1}{2} & S_{0}^{\prime \prime}
\end{array}\right\}\right.} \\
& \left.\left\{\begin{array}{ccc}
L^{\prime} & L^{\prime \prime} & 1 \\
l & l & L_{0}^{\prime \prime}
\end{array}\right\}\left\{\begin{array}{ccc}
S^{\prime \prime} & S & \kappa \\
\frac{1}{2} & \frac{1}{2} & S_{0}^{\prime \prime}
\end{array}\right\}\left\{\begin{array}{ccc}
L^{\prime \prime} & L & k \\
l & l & L_{0}^{\prime \prime}
\end{array}\right\}\right]
\end{aligned}
$$

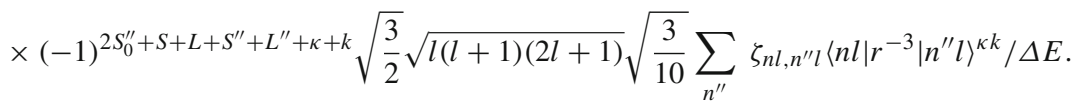

\section{A. $3 n l^{N} n_{1} l_{1}^{N_{1}}$ configuration}

The states $\psi$ and $\psi^{\prime}$ for $n l^{N} n_{1} l_{1}^{N_{1}}$ configuration are defined as follows: $\psi=n l^{N} \alpha_{1} S_{1} L_{1}, n_{1} l_{1}^{N_{1}} \alpha_{2} S_{2} L_{2} ; S L, \psi^{\prime}=n l^{N} \alpha_{1}^{\prime} S_{1}^{\prime} L_{1}^{\prime}, n_{1} l_{1}^{N_{1}} \alpha_{2}^{\prime} S_{2}^{\prime} L_{2}^{\prime} ; S^{\prime} L^{\prime}$.

In this case, the perturbing virtual states are defined as follows: $\psi^{\prime \prime}=\left(n l^{N-1} \alpha_{0}^{\prime \prime} S_{0}^{\prime \prime} L_{0}^{\prime \prime}, n^{\prime \prime} l^{\prime \prime}\right) S_{1}^{\prime \prime} L_{1}^{\prime \prime}, n_{1} l_{1}^{N_{1}} \alpha_{2}^{\prime \prime} S_{2}^{\prime \prime} L_{2}^{\prime \prime} ; S^{\prime \prime} L^{\prime \prime}$ and $\kappa k=11$ or 13 .

$$
\begin{aligned}
& -\sum_{\psi^{\prime \prime}}\left[\left\langle\psi\left\|\mathbf{H}_{\mathrm{so}}\right\| \psi^{\prime \prime}\right\rangle \times\left\langle\psi^{\prime \prime}\left\|\mathbf{t}^{\kappa k}\right\| \psi^{\prime}\right\rangle+\left\langle\psi\left\|\mathbf{t}^{\kappa k}\right\| \psi^{\prime \prime}\right\rangle \times\left\langle\psi^{\prime \prime}\left\|\mathbf{H}_{\mathrm{so}}\right\| \psi^{\prime}\right\rangle\right] / \Delta E \\
& =\sum_{\alpha_{0}^{\prime \prime} S_{0}^{\prime \prime} L_{0}^{\prime \prime}, S_{1}^{\prime \prime} L_{1}^{\prime \prime}, S^{\prime \prime} L^{\prime \prime}} \delta\left(l, l^{\prime \prime}\right) \delta\left(\alpha_{2} S_{2} L_{2}, \alpha_{2}^{\prime \prime} S_{2}^{\prime \prime} L_{2}^{\prime \prime}\right) \delta\left(\alpha_{2}^{\prime \prime} S_{2}^{\prime \prime} L_{2}^{\prime \prime}, \alpha_{2}^{\prime} S_{2}^{\prime} L_{2}^{\prime}\right) \\
& \times N\left(n l ^ { N } \alpha _ { 1 } S _ { 1 } L _ { 1 } \{ | n l ^ { N - 1 } \alpha _ { 0 } ^ { \prime \prime } S _ { 0 } ^ { \prime \prime } L _ { 0 } ^ { \prime \prime } ) \left(n l^{N} \alpha_{1}^{\prime} S_{1}^{\prime} L_{1}^{\prime}\left\{\mid n l^{N-1} \alpha_{0}^{\prime \prime} S_{0}^{\prime \prime} L_{0}^{\prime \prime}\right)\right.\right. \\
& \times(-1)^{2 S_{1}+2 S_{2}+S^{\prime \prime}+L^{\prime \prime}+2 S_{0}^{\prime \prime}+2 S_{1}^{\prime \prime}+S^{\prime}+L^{\prime}}\left[S, L, S^{\prime}, L^{\prime}\right]^{1 / 2}\left[S_{1}, L_{1}, S_{1}^{\prime}, L_{1}^{\prime}\right]^{1 / 2}\left[S^{\prime \prime}, L^{\prime \prime}\right]\left[S_{1}^{\prime \prime}, L_{1}^{\prime \prime}\right] \\
& \times\left[\left\{\begin{array}{ccc}
S & S^{\prime \prime} & 1 \\
S_{1}^{\prime \prime} & S_{1} & S_{2}
\end{array}\right\}\left\{\begin{array}{ccc}
L & L^{\prime \prime} & 1 \\
L_{1}^{\prime \prime} & L_{1} & L_{2}
\end{array}\right\}\left\{\begin{array}{ccc}
S_{1} & S_{1}^{\prime \prime} & 1 \\
\frac{1}{2} & \frac{1}{2} & S_{0}^{\prime \prime}
\end{array}\right\}\right. \\
& \left\{\begin{array}{ccc}
L_{1} & L_{1}^{\prime \prime} & 1 \\
l & l & L_{0}^{\prime \prime}
\end{array}\right\}\left\{\begin{array}{ccc}
S^{\prime \prime} & S^{\prime} & \kappa \\
S_{1}^{\prime} & S_{1}^{\prime \prime} & S_{2}^{\prime}
\end{array}\right\}\left\{\begin{array}{ccc}
L^{\prime \prime} & L^{\prime} & k \\
L_{1}^{\prime} & L_{1}^{\prime \prime} & L_{2}^{\prime}
\end{array}\right\}\left\{\begin{array}{ccc}
S_{1}^{\prime \prime} & S_{1}^{\prime} & \kappa \\
\frac{1}{2} & \frac{1}{2} & S_{0}^{\prime \prime}
\end{array}\right\}\left\{\begin{array}{ccc}
L_{1}^{\prime \prime} & L_{1}^{\prime} & k \\
l & l & L_{0}^{\prime \prime}
\end{array}\right\} \\
& +\left\{\begin{array}{ccc}
S & S^{\prime \prime} & \kappa \\
S_{1}^{\prime \prime} & S_{1} & S_{2}
\end{array}\right\}\left\{\begin{array}{ccc}
L & L^{\prime \prime} & k \\
L_{1}^{\prime \prime} & L_{1} & L_{2}
\end{array}\right\}\left\{\begin{array}{ccc}
S_{1} & S_{1}^{\prime \prime} & \kappa \\
\frac{1}{2} & \frac{1}{2} & S_{0}^{\prime \prime}
\end{array}\right\}\left\{\begin{array}{ccc}
L_{1} & L_{1}^{\prime \prime} & k \\
l & l & L_{0}^{\prime \prime}
\end{array}\right\} \\
& \left.\left\{\begin{array}{ccc}
S^{\prime \prime} & S^{\prime} & 1 \\
S_{1}^{\prime} & S_{1}^{\prime \prime} & S_{2}^{\prime}
\end{array}\right\}\left\{\begin{array}{ccc}
L^{\prime \prime} & L^{\prime} & 1 \\
L_{1}^{\prime} & L_{1}^{\prime \prime} & L_{2}^{\prime}
\end{array}\right\}\left\{\begin{array}{ccc}
S_{1}^{\prime \prime} & S_{1}^{\prime} & 1 \\
\frac{1}{2} & \frac{1}{2} & S_{0}^{\prime \prime}
\end{array}\right\}\left\{\begin{array}{ccc}
L_{1}^{\prime \prime} & L_{1}^{\prime} & 1 \\
l & l & L_{0}^{\prime \prime}
\end{array}\right\}\right] \\
& \times \sqrt{\frac{3}{2}} \sqrt{l(l+1)(2 l+1)} \sqrt{\frac{3}{10}} \sum_{n^{\prime \prime}} \zeta_{n l, n^{\prime \prime} l}\left\langle n l\left|r^{-3}\right| n^{\prime \prime} l\right\rangle^{\kappa k} / \Delta E \text {. }
\end{aligned}
$$


A.4 $n l^{N} n_{1} l_{1}^{N_{1}} n_{2} l_{2}$ configuration

The states $\psi$ and $\psi^{\prime}$ for $n l^{N} n_{1} l_{1}^{N_{1}} n_{2} l_{2}$ configuration are defined as follows:

$$
\begin{aligned}
& \psi=n l^{N} \alpha_{1} S_{1} L_{1},\left(n_{1} l_{1}^{N_{1}} \alpha_{2} S_{2} L_{2}, n_{2} l_{2}\right) S_{3} L_{3} ; S L, \\
& \psi^{\prime}=n l^{N} \alpha_{1}^{\prime} S_{1}^{\prime} L_{1}^{\prime},\left(n_{1} l_{1}^{N_{1}} \alpha_{2}^{\prime} S_{2}^{\prime} L_{2}^{\prime}, n_{2} l_{2}\right) S_{3}^{\prime} L_{3}^{\prime} ; S^{\prime} L^{\prime} .
\end{aligned}
$$

In this case, the perturbing virtual states are defined as follows: $\psi^{\prime \prime}=\left(n l^{N-1} \alpha_{0}^{\prime \prime} S_{0}^{\prime \prime} L_{0}^{\prime \prime}, n^{\prime \prime} l^{\prime \prime}\right) S_{1}^{\prime \prime} L_{1}^{\prime \prime},\left(n_{1} l_{1}^{N_{1}} \alpha_{2}^{\prime \prime} S_{2}^{\prime \prime} L_{2}^{\prime \prime}, n_{2} l_{2}\right) S_{3}^{\prime \prime} L_{3}^{\prime \prime} ; S^{\prime \prime} L^{\prime \prime}$ and $\kappa k=11$ or 13 .

$$
\begin{aligned}
& -\sum_{\psi^{\prime \prime}}\left[\left\langle\psi\left\|\mathbf{H}_{\mathrm{so}}\right\| \psi^{\prime \prime}\right\rangle \times\left\langle\psi^{\prime \prime}\left\|\mathbf{t}^{\kappa k}\right\| \psi^{\prime}\right\rangle+\left\langle\psi\left\|\mathbf{t}^{\kappa k}\right\| \psi^{\prime \prime}\right\rangle \times\left\langle\psi^{\prime \prime}\left\|\mathbf{H}_{\mathrm{so}}\right\| \psi^{\prime}\right\rangle\right] / \Delta E \\
& =\sum_{\alpha_{0}^{\prime \prime} S_{0}^{\prime \prime} L_{0}^{\prime \prime}, S_{1}^{\prime \prime} L_{1}^{\prime \prime}, S^{\prime \prime} L^{\prime \prime}} \delta\left(l, l^{\prime \prime}\right) \delta\left(\alpha_{2} S_{2} L_{2}, \alpha_{2}^{\prime \prime} S_{2}^{\prime \prime} L_{2}^{\prime \prime}\right) \\
& \delta\left(\alpha_{2}^{\prime \prime} S_{2}^{\prime \prime} L_{2}^{\prime \prime}, \alpha_{2}^{\prime} S_{2}^{\prime} L_{2}^{\prime}\right) \delta\left(S_{3} L_{3}, S_{3}^{\prime \prime} L_{3}^{\prime \prime}\right) \delta\left(S_{3}^{\prime \prime} L_{3}^{\prime \prime}, S_{3}^{\prime} L_{3}^{\prime}\right) \\
& \times N\left(n l ^ { N } \alpha _ { 1 } S _ { 1 } L _ { 1 } \{ | n l ^ { N - 1 } \alpha _ { 0 } ^ { \prime \prime } S _ { 0 } ^ { \prime \prime } L _ { 0 } ^ { \prime \prime } ) \left(n l^{N} \alpha_{1}^{\prime} S_{1}^{\prime} L_{1}^{\prime}\left\{\mid n l^{N-1} \alpha_{0}^{\prime \prime} S_{0}^{\prime \prime} L_{0}^{\prime \prime}\right)\right.\right. \\
& \times(-1)^{2 S_{1}+2 S_{3}+S^{\prime \prime}+L^{\prime \prime}+2 S_{0}^{\prime \prime}+2 S_{1}^{\prime \prime}+S^{\prime}+L^{\prime}}\left[S, L, S^{\prime}, L^{\prime}\right]^{1 / 2}\left[S_{1}, L_{1}, S_{1}^{\prime}, L_{1}^{\prime}\right]^{1 / 2}\left[S^{\prime \prime}, L^{\prime \prime}\right]\left[S_{1}^{\prime \prime}, L_{1}^{\prime \prime}\right] \\
& \times\left[\left\{\begin{array}{ccc}
S & S^{\prime \prime} & 1 \\
S_{1}^{\prime \prime} & S_{1} & S_{3}
\end{array}\right\}\left\{\begin{array}{ccc}
L & L^{\prime \prime} & 1 \\
L_{1}^{\prime \prime} & L_{1} & L_{3}
\end{array}\right\}\left\{\begin{array}{lll}
S^{\prime \prime} & S^{\prime} & \kappa \\
S_{1}^{\prime} & S_{1}^{\prime \prime} & S_{3}
\end{array}\right\}\left\{\begin{array}{lll}
L^{\prime \prime} & L^{\prime} & k \\
L_{1}^{\prime} & L_{1}^{\prime \prime} & L_{3}
\end{array}\right\}\right. \\
& \left\{\begin{array}{ccc}
S_{1} & S_{1}^{\prime \prime} & 1 \\
\frac{1}{2} & \frac{1}{2} & S_{0}^{\prime \prime}
\end{array}\right\}\left\{\begin{array}{ccc}
L_{1} & L_{1}^{\prime \prime} & 1 \\
l & l & L_{0}^{\prime \prime}
\end{array}\right\}\left\{\begin{array}{ccc}
S_{1}^{\prime \prime} & S_{1}^{\prime} & \kappa \\
\frac{1}{2} & \frac{1}{2} & S_{0}^{\prime \prime}
\end{array}\right\}\left\{\begin{array}{ccc}
L_{1}^{\prime \prime} & L_{1}^{\prime} & k \\
l & l & L_{0}^{\prime \prime}
\end{array}\right\} \\
& +\left\{\begin{array}{ccc}
S & S^{\prime \prime} & \kappa \\
S_{1}^{\prime \prime} & S_{1} & S_{3}
\end{array}\right\}\left\{\begin{array}{ccc}
L & L^{\prime \prime} & k \\
L_{1}^{\prime \prime} & L_{1} & L_{3}
\end{array}\right\}\left\{\begin{array}{lll}
S^{\prime \prime} & S^{\prime} & 1 \\
S_{1}^{\prime} & S_{1}^{\prime \prime} & S_{3}
\end{array}\right\}\left\{\begin{array}{lll}
L^{\prime \prime} & L^{\prime} & 1 \\
L_{1}^{\prime} & L_{1}^{\prime \prime} & L_{3}
\end{array}\right\} \\
& \left.\left\{\begin{array}{ccc}
S_{1}^{\prime} & S_{1}^{\prime \prime} & 1 \\
\frac{1}{2} & \frac{1}{2} & S_{0}^{\prime \prime}
\end{array}\right\}\left\{\begin{array}{ccc}
L_{1}^{\prime} & L_{1}^{\prime \prime} & 1 \\
l & l & L_{0}^{\prime \prime}
\end{array}\right\}\left\{\begin{array}{ccc}
S_{1}^{\prime \prime} & S_{1} & \kappa \\
\frac{1}{2} & \frac{1}{2} & S_{0}^{\prime \prime}
\end{array}\right\}\left\{\begin{array}{ccc}
L_{1}^{\prime \prime} & L_{1} & k \\
l & l & L_{0}^{\prime \prime}
\end{array}\right\}\right] \\
& \times \sqrt{\frac{3}{2}} \sqrt{l(l+1)(2 l+1)} \sqrt{\frac{3}{10}} \sum_{n^{\prime \prime}} \zeta_{n l, n^{\prime \prime} l}\left\langle n l\left|r^{-3}\right| n^{\prime \prime} l\right\rangle^{\kappa k} / \Delta E \text {. }
\end{aligned}
$$

\section{References}

1. D. Yordanov, L. Rodriguez, D. Balabanski, J. Bieron, M. Bissell, K. Blaum, B. Cheal, J. Ekman, G. Gaigalas, R. Garcia Ruiz, G. Georgiev, W. Gins, M. Godefroid, C. Gorges, Z. Harman, H. Heylen, P. Jönsson, A. Kanellakopoulos, S. Kaufmann, C. Keitel, V. Lagaki, S. Lechner, B. Maass, S. MalbrunotEttenauer, W. Nazarewicz, R. Neugart, G. Neyens, W. Noertershaeuser, N. Oreshkina, A. Papoulia, P. Pyykkö, P.G. Reinhard, S. Sailer, R. Sanchez, S. Schiffmann, S. Schmidt, L. Wehner, C. Wraith, L. Xie, Z. Xu, X. Yang, Commun. Phys. 3, 1 (2020). https://doi.org/10.1038/s42005-020-0348-9

2. N. Stone, At. Data Nucl. Data Tables 111-112, 1 (2016). https://doi.org/10.1016/j.adt.2015.12.002

3. P. Raghavan, At. Data Nucl. Data Tables 42(2), 189 (1989). https://doi.org/10.1016/0092640X(89)90008-9

4. N. Stone, At. Data Nucl. Data Tables 90(1), 75 (2005). https://doi.org/10.1016/j.adt.2005.04.001

5. P. Pyykkö, Mol. Phys. 116(10), 1328 (2018). https://doi.org/10.1080/00268976.2018.1426131

6. W.J. Childs, Phys. Rev. A 4, 439 (1971). https://doi.org/10.1103/PhysRevA.4.439

7. M. Anselment, K. Bekk, A. Hanser, H. Hoeffgen, G. Meisel, S. Göring, H. Rebel, G. Schatz, Phys. Rev. C 34, 1052 (1986). https://doi.org/10.1103/PhysRevC.34.1052

8. D. Berdichevsky, R. Fleming, D.W.L. Sprung, F. Tondeur, Z. Phys. A. 329(4), 393 (1988). https://doi. org/10.1007/BF01294344

9. F. Le Blanc, L. Cabaret, J.E. Crawford, S. Essabaa, V. Fedoseyev, W. Geithner, J. Genevey, M. Girod, R. Horn, G. Huber, S. Kappertz, J. Lassen, J.K.P. Lee, G. Le Scornet, V. Mishin, R. Neugart, J. Obert, J. Oms, 
A. Ouchrif, S. Peru, J. Pinard, H. Ravn, B. Roussière, J. Sauvage, D. Verney, the Isolde Collaboration, Eur. Phys. J. A 15(1), 49 (2002). https://doi.org/10.1140/epja/i2001-10225-9

10. F.L. Blanc, L. Cabaret, E. Cottereau, J.E. Crawford, S. Essabaa, J. Genevey, R. Horn, G. Huber, J. Lassen, J.K.P. Lee, G.L. Scornet, J. Lettry, J. Obert, J. Oms, A. Ouchrif, J. Pinard, H. Ravn, B. Roussière, J. Sauvage, D. Verney, Phys. Rev. C 72, 034305 (2005). https://doi.org/10.1103/PhysRevC.72.034305

11. G. Barone, R. Mastalerz, M. Reiher, R. Lindh, J. Phys. Chem. A 112(7), 1666 (2008). https://doi.org/10. 1021/jp710388t

12. J.M. Allmond, A.E. Stuchbery, A. Galindo-Uribarri, E. Padilla-Rodal, D.C. Radford, J.C. Batchelder, C.R. Bingham, M.E. Howard, J.F. Liang, B. Manning, S.D. Pain, N.J. Stone, R.L. Varner, C.H. Yu, Phys. Rev. C 92, 041303 (2015). https://doi.org/10.1103/PhysRevC.92.041303

13. P. Jönsson, G. Gaigalas, J. Bieroń, C.F. Fischer, I.P. Grant, Comput. Phys. Commun. 184(9), 2197 (2013). https://doi.org/10.1016/j.cpc.2013.02.016

14. C. Froese Fischer, G. Gaigalas, P. Jönsson, J. Bieroń, Comput. Phys. Commun. 237, 184 (2019). https:// doi.org/10.1016/j.cpc.2018.10.032

15. I. Tupitsyn, A. Loginov, Opt. Spectrosc. 94, 319 (2003). https://doi.org/10.1134/1.1563671

16. A. Papoulia, S. Schiffmann, J. Bieron, G. Gaigalas, M. Godefroid, Z. Harman, P. Jönsson, N.S. Oreshkina, P. Pyykkö, I.I. Tupitsyn, Phys. Rev. A 103, 022815 (2021). https://doi.org/10.1103/PhysRevA.103.022815

17. J. Eberz, U. Dinger, G. Huber, H. Lochmann, R. Menges, G. Ulm, R. Kirchner, O. Klepper, T.U. Kühl, D. Marx, Z. Phys. A 326(2), 121 (1987). https://doi.org/10.1007/BF01283586

18. M. Elantkowska, J. Ruczkowski, S. Wilman, J. Dembczyński, At. Data Nucl. Data Tables 135-136, 101342 (2020). https://doi.org/10.1016/j.adt.2020.101342

19. J. Ruczkowski, M. Elantkowska, J. Dembczyński, J. Quant. Spectrosc. Radiat. Transf. 145, 20 (2014). https://doi.org/10.1016/j.jqsrt.2014.04.018

20. J. Ruczkowski, M. Elantkowska, J. Dembczyński, J. Quant. Spectrosc. Radiat. Transf. 149, 168 (2014). https://doi.org/10.1016/j.jqsrt.2014.08.010

21. J. Ruczkowski, M. Elantkowska, J. Dembczyński, J. Quant. Spectrosc. Radiat. Transf. 170, 106 (2016). https://doi.org/10.1016/j.jqsrt.2015.10.023

22. J. Dembczyński, G. Szawioła, M. Elantkowska, E. Stachowska, J. Ruczkowski, Phys. Scr. 54(5), 444 (1996). https://doi.org/10.1088/0031-8949/54/5/004

23. M. Elantkowska, J. Ruczkowski, J. Dembczyński, Phys. Scr. 59(1), 49 (1999). https://doi.org/10.1238/ Physica.Regular.059a00049

24. M. Elantkowska, J. Ruczkowski, J. Dembczyński, Eur. Phys. J. Plus 130, 14 (2015). https://doi.org/10. 1140/epjp/i2015-15014-8

25. M. Elantkowska, J. Ruczkowski, J. Dembczyński, Eur. Phys. J. Plus 130, 15 (2015). https://doi.org/10. 1140/epjp/i2015-15015-7

26. M. Elantkowska, J. Ruczkowski, J. Dembczyński, Eur. Phys. J. Plus 130, 83 (2015). https://doi.org/10. 1140/epjp/i2015-15083-7

27. M. Elantkowska, J. Ruczkowski, J. Dembczyński, Eur. Phys. J. Plus 130, 170 (2015). https://doi.org/10. 1140/epjp/i2015-15170-9

28. M. Elantkowska, J. Ruczkowski, J. Dembczyński, Eur. Phys. J. Plus 131(2), 47 (2016). https://doi.org/ 10.1140/epjp/i2016-16047-1

29. P.G.H. Sandars, J. Beck, Proc. R. Soc. Lond. Ser. A Math. Phys. Sci. 289(1416), 97 (1965). https://doi. org/10.1098/rspa.1965.0251

30. J. Dembczyński, M. Elantkowska, B. Furmann, J. Ruczkowski, D. Stefańska, J. Phys. B At. Mol. Opt. Phys. 43(6), 065001 (2010). https://doi.org/10.1088/0953-4075/43/6/065001

31. M. Elantkowska, J. Ruczkowski, J. Dembczyński, Eur. Phys. J. Plus 131(12), 429 (2016). https://doi.org/ 10.1140/epjp/i2016-16429-3

32. S. Feneuille, L. Armstrong, Phys. Rev. A 8, 1173 (1973). https://doi.org/10.1103/PhysRevA.8.1173

33. L. Armstrong Jr., Theory of the Hyperfine Structure of Free Atoms (Willey-Interscience, New York, 1971)

34. S. Feneuille, J. Phys. France 28(1), 61 (1967). https://doi.org/10.1051/jphys:0196700280106100

35. M.G.H. Gustavsson, A.M. Mårtensson-Pendrill, Phys. Rev. A 58, 3611 (1998). https://doi.org/10.1103/ PhysRevA.58.3611

36. W. Makulski, K. Jackowski, A. Antušek, M. Jaszuński, J. Phys. Chem. A 110, 11462 (2006). https://doi. org/10.1021/jp062557s

37. W. Makulski, J. Mol. Struct. 1017, 45 (2012). https://doi.org/10.1016/j.molstruc.2012.02.072

38. E. Malkin, S. Komorovsky, M. Repisky, T.B. Demissie, K. Ruud, J. Phys. Chem. Lett. 4, 459 (2013). https://doi.org/10.1021/jz302146m

39. I. Lindgren, A. Rosén, in Case Studies in Atomic Physics, ed. by E. McDaniel, M. McDowell (Elsevier, 1975), pp. 93-196. https://doi.org/10.1016/B978-0-7204-0331-2.50007-X

40. J. Bieroń, P. Pyykkö, Phys. Rev. Lett. 87, 133003 (2001). https://doi.org/10.1103/PhysRevLett.87.133003 
41. T.Q. Teodoro, R.L.A. Haiduke, Phys. Rev. A 88, 052504 (2013). https://doi.org/10.1103/PhysRevA.88. 052504 